\title{
Set4 coordinates the activity of histone deacetylases and regulates stress- responsive gene expression within subtelomeric regions in yeast
}

Yogita Jethmalani ${ }^{1}$, Khoa $\operatorname{Tran}^{1,3}$, Deepika Jaiswal ${ }^{1}$, Meagan Jezek ${ }^{1}$, Mark Ramos ${ }^{2}$, Shandon Amos ${ }^{1}$, Eric Joshua Garcia ${ }^{1}$, Maraki Y. Negesse ${ }^{1}$, Winny Sun ${ }^{1}$, DoHwan Park $^{2}$ and Erin M. Green ${ }^{1, *}$

${ }^{1}$ Department of Biological Sciences, University of Maryland Baltimore County, Baltimore, Maryland 21250

${ }^{2}$ Department of Mathematics and Statistics, University of Maryland Baltimore County, Baltimore, Maryland 21250

${ }^{3}$ Current address: Laboratory of Cellular and Developmental Biology, National Institute of Diabetes and Digestive and Kidney Disorders, National Institutes of Health, Bethesda, MD 20892

${ }^{*}$ Corresponding author: Erin M. Green; egreen@umbc.edu

Keywords: chromatin, Set4, stress responses, telomeres, histone deacetylases, hypoxia, oxidative stress, yeast 


\section{Abstract}

The yeast chromatin protein Set4 is a member of the Set3-subfamily of SET domain proteins which play critical roles in the regulation of gene expression in diverse developmental and environmental contexts, although they appear to lack methyltransferase activity. The molecular functions of Set4 are relatively unexplored, likely due to its low abundance in standard growth conditions. We previously reported that Set4 promotes survival during oxidative stress and regulates expression of stress response genes via stress-dependent chromatin localization. In this study, global gene expression analysis and investigation of histone modification status has revealed a role for Set4 in maintaining gene repressive mechanisms within yeast subtelomeres under both normal and stress conditions. We show that Set4 works in a partially overlapping pathway to the SIR complex and the histone deacetylase Rpd3 to maintain proper levels of histone acetylation and expression of stress response genes encoded in subtelomeres. This role for Set4 is particularly critical for cells under hypoxic conditions, and the loss of Set4 decreases cell fitness and cell wall integrity in hypoxia. These findings uncover a new regulator of subtelomeric chromatin that is key to stress defense pathways and demonstrate a function for yeast Set4 in regulating repressive, heterochromatin-like environments. 


\section{Introduction}

The regulation of gene expression in response to changing environmental signals is dependent on a diverse set of chromatin-binding proteins, including transcription factors, histone-modifying enzymes, and chromatin remodeling complexes. Proteins containing a SET domain are well-established regulators of gene expression primarily through the catalysis of methylation of lysine residues within histones $(1,2)$, though SET domain proteins also methylate non-histone substrates $(3,4)$. A subfamily of SET domain proteins, often referred to as the Set3 subfamily, is characterized by divergent SET domains which appear to lack methyltransferase activity due to amino acid substitutions at key substrate binding interfaces $(5,6)$. This subfamily includes the Saccharomyces cerevisiae paralogs Set3 and Set4, SET-9 and SET-26 from Caenorhabditis elegans, UpSET from Drosophila melanogaster, and the mammalian proteins MLL5 and SETD5 (7). Instead of directly catalyzing lysine methylation at chromatin, the Set3 subfamily of proteins are thought to regulate gene expression by binding to and regulating histone deacetylases (HDACs) at chromatin $(8,9)$.

Our previous work identified a role for the yeast protein Set4, a paralog to Set3, in protecting cells during oxidative stress, primarily through gene expression regulation of stress response genes (10). Set4 is lowly expressed in yeast cells under standard laboratory growth conditions, although deletion of SET4 increases sensitivity to acute oxidative stress and alters gene expression patterns (7,10-12), indicating a biological function for Set4 even at low abundance. Expression of SET4 appears to be stressregulated, as the transcript and protein levels increase in low oxygen, including hypoxic or anaerobic conditions $(13,14)$. Other work has also implicated Set4 in the regulation of gene expression during hypoxia (14), where it was shown to repress ergosterol biosynthetic genes together with the transcriptional repressor Hap1 through the inhibition of the sterol-responsive activator Upc2 (14).

The subtelomeric regions of $S$. cerevisiae and other fungi, such as Candida glabrata, harbor many stress-response genes, particularly those that control adhesion, filamentation, and adaptation to anaerobic environments $(15,16)$. Gene expression within subtelomeres is generally very low (17) due to regional silencing mechanisms, such as by the SIR histone deacetylase complex, and other transcriptional repressors (18). In other systems, Set3- and Set4-related proteins have been shown to maintain heterochromatic or repressive chromatin environments, including the fission yeast ortholog Set3 (19), the fly ortholog UpSET which interacts with the Rpd3/Sin3 deacetylase complex $(20,21)$ and the $C$. elegans orthologs SET-9 and SET-26 which restrict spreading of $\mathrm{H} 3 \mathrm{~K} 4 \mathrm{me} 3-\mathrm{demarcated}$ regions to regulate expression of germlinespecific genes (22). While Set3 in budding yeast is critical for gene repression in multiple contexts, it is not known to have a specific role in maintaining silent chromatin states such as at subtelomeres, the mating type locus, or ribosomal DNA (rDNA) locus $(8,9,23)$. Given the structural similarities between yeast Set3 and Set4 and orthologous proteins (7), we hypothesized that Set4 may function in regulating silent chromatin regions in yeast, especially since genes required for multiple stress response pathways are found within silent regions such as subtelomeres (18). Here, we demonstrate that 
Set4 calibrates gene expression within yeast subtelomeres under both normal and stress conditions and contributes to cell fitness and cell wall integrity in hypoxic conditions. In hypoxia, Set4 promotes subtelomeric chromatin binding of the HDACs Sir2 and Rpd3, which have previously been implicated in the regulation of stress response and subtelomeric genes (17,24-29). This maintains proper levels of histone acetylation and fine-tunes expression levels of genes within the subtelomere. These data uncover a key function of Set4 in controlling subtelomeric chromatin to coordinate proper gene expression in response to stress. Furthermore, our results indicate that while this regulatory role of Set4 is performed under non-stress conditions, it becomes critical for cells in response to certain environmental signals, including oxidative stress and limiting oxygen.

\section{Materials and Methods}

\section{Yeast strains and growth conditions}

The genotypes for all Saccharomyces cerevisiae strains used in this study are listed in Table S1. Strains carrying gene deletions were made using targeted PCR cassettes amplified from the pFA6a vector series (30). Double mutant strains were isolated following haploid mating, sporulation and tetrad dissection. All strain genotypes were confirmed by growth on the appropriate selective media and colony PCR using primers specific to individual gene deletions or epitope tag insertions. Standard media conditions for rich media (YPD; 1\% yeast extract, 1\% peptone, 2\% dextrose) and synthetic complete (SC) or dropout media (US Biological) were used as necessary. For all growth assays, gene expression and chromatin immunoprecipitation experiments, yeast cultures were diluted and grown in appropriate media overnight to mid-log phase $\left(\mathrm{OD}_{600} \sim 0.4-0.8\right)$ at $30^{\circ} \mathrm{C}$. For hypoxic growth, the culture flasks were placed in $\mathrm{BD}$ GasPak EZ anaerobe pouch system and incubated at $30^{\circ} \mathrm{C}$. For hydrogen peroxidetreated cultures, cells were grown to mid-log phase $\left(O D_{600} \sim 0.6-0.8\right)$ and then treated with $0.4 \mathrm{mM} \mathrm{H}_{2} \mathrm{O}_{2}$ for $30 \mathrm{~min}$ (12).

\section{RNA Sequencing}

Total RNA was extracted from yeast cells and subjected to Illumina-based RNAsequencing at Genewiz (South Plainfield, NJ). Differential gene expression analysis was performed as previously described $(31,32)$. Briefly, read quality control was analyzed using FastQC and adaptor removal and read trimming were performed with Trimommatic v.0.36 (33). Reads were mapped to the $S$. cerevisiae reference genome using the STAR aligner v.2.5.2b and Subread package v.1.5.2 was used for calculating gene hit counts (34). The data were normalized and log-fold change values were determined using DESeq2 (35). The raw and processed data for RNA-sequencing experiments are available on the Gene Expression Omnibus database at accession number GSE173901.

\section{Differential gene expression significance testing}

For testing the significance of gene expression changes, we use a hybrid of two existing methods depending on the applicability of zero assumption in Efron (36): the center of 
the observed $\log _{2}$ fold-change (log FC) values consists of non-differentially expressed genes. One method is the local false discovery rate procedure (local FDR) which estimates the distribution of the non-differentially expressed genes based on zero assumptions instead of using the standard normal distribution. It can be a more powerful test when zero assumption holds: log FC values showed little change at most genes with small groups of up- and down-regulated genes exhibiting the most change (Figure S1). Local FDR analysis was employed to identify differentially-expressed genes at FDR $\leq 0.05$ for datasets comparing wildtype and set4 $\Delta$ cells. The false discovery rate is computed from these estimates and is controlled to be less than $5 \%$. The method is implemented through the locfdr package in $\mathrm{R}$ (37).

The other method used for datasets comparing expression differences between aerobic and hypoxic conditions is the test procedure in DESeq2 after filtering absolute value of $\log \mathrm{FC}>1$, the Wald test $\mathrm{p}$-values which are adjusted (padjust) for multiple testing using the procedure of Benjamini and Hochberg (38). Since the test in DESeq2 does not need the structure assumption such as zero assumption, we applied it when there was large variability of log FC values and more of the non-differentially expressed genes spread out due to the discrepancy resulting from a larger number of up- and down-regulated genes. Padjust $\leq 0.05$ are selected to be differentially expressed genes which represents FDR $\leq 0.05$.

Gene ontology analysis was performed using the GO term function in Yeastmine and the GO term slim mapper through the Saccharomyces Genome Database. Telomere enrichment was determined by identifying the number of genes within $40 \mathrm{~kb}$ of the telomere end in each dataset analyzed and using a hypergeometric test to determine significance of enrichment and fold-enrichment over expected based on the total number of genes within subtelomeres in the genome.

\section{Gene expression analysis by quantitative reverse transcriptase PCR}

Total RNA was extracted using $1.5 \mathrm{ml}$ of mid log phase culture of yeast cells $\left(\mathrm{OD}_{600}\right.$ 0.6-0.8) under different growth conditions. Masterpure Yeast RNA purification kit (Epicentre) was used to extract the RNA by following the manufacturer's instructions. Turbo DNA-free kit (Ambion) was used to eliminate genomic DNA from the samples. cDNA was generated from $1 \mu \mathrm{g}$ of total RNA using qMax cDNA synthesis kit (Accuris) containing both oligo $\mathrm{dT}$ and random hexamers for priming reverse transcription of mRNA. For quantitative PCR (qPCR) to check transcript levels $0.5 \mu \mathrm{l}$ of cDNA was added to 1X qMax Green Low ROX qPCR mix (Accuris) with the appropriate gene specific primers (Table S2) in a $10 \mu$ reaction. Real-time amplification was performed on a Bio-Rad CFX384 Real-time Detection System. Three technical replicates were performed for each reaction and a minimum of three biological replicates was performed for each experiment. Relative gene expression values were normalized to the control gene TFC1, whose expression has been shown to be stable under different growth conditions (39).

\section{Spot assays}

For the telomere position effect spot assay, strains integrated with the URA3 gene at TEL VIIL were used (see Table S1; kindly provided by Paul Kaufman). Gene knockouts were created using insertion of targeted PCR cassettes amplified from the pFA6a vector 
series (30). Cells were grown overnight in YPD medium at $30^{\circ} \mathrm{C}$ and $0.1 \mathrm{OD}$ units of the cultures were serially diluted and spotted on YPD plates (control) and 5-fluoroorotic acid (5-FOA) plates. The plates were observed and imaged for two days to analyze the growth pattern. For growth analysis of single and double mutant strains under aerobic and hypoxic conditions, yeast strains were grown overnight in YPD, diluted to $\mathrm{OD}_{600}$ 0.2 the next day, and grown to log phase. $0.1 \mathrm{OD}_{600}$ units of the culture were serially diluted and spotted on YPD plates. For hypoxic conditions, the plates were incubated in BD GasPak EZ anaerobe pouches. The plates were observed and imaged for two days for aerobic conditions and eight days for hypoxic conditions.

\section{Telomere Southern Blot}

Whole cell extract from wildtype and set4 $\Delta$ strains was made by bead beating in phenolchloroform-isoamyl alcohol. The extract was treated with RNase A and DNA was precipitated using ethanol. Genomic DNA was digested using the restriction enzyme Xhol, extracted with phenol-chloroform-isoamyl alcohol, and precipitated with ethanol. Digested DNA was subjected to electrophoresis on an $0.8 \%$ agarose gel in $0.5 \mathrm{X}$ TBE, the DNA was denatured in-gel and transferred onto a Hybond $\mathrm{N}+$ nylon membrane (Amersham). The membrane was hybridized with a biotin-conjugated telomere probe (5'-biotin-CACACCCACACCCACACC-3') and was imaged using a Chemiluminescent Nucleic Acid Detection Module Kit (Thermo Scientific) and a Li-Cor C-DiGit Chemiluminescent Western Blot scanner.

\section{Zymolyase sensitivity assay}

WT (yEG001) and set4A (yEG322) were diluted and grown overnight to mid-log phase $\left(\mathrm{OD}_{600} \sim 0.4-0.8\right)$ in aerobic or hypoxic conditions. Cells were collected and resuspended in $1 \mathrm{~mL}$ sorbitol buffer (1.2 M Sorbitol, $0.1 \mathrm{M} \mathrm{KP04} \mathrm{pH} 7.5)$ and $5 \mu \mathrm{l}$ of 2mercaptoethanol and $5 \mu \mathrm{l}$ of $10 \mathrm{mg} / \mathrm{mL}$ 100T Zymolyase was added. Cells were incubated at room temperature, with occasional rocking, and the $\mathrm{OD}_{600}$ was measured every 5 minutes in 1\% SDS. Time $50 \%$ oD was determined as the time elapsed for the cultures to reach $50 \%$ of the starting $\mathrm{OD}_{600}$, indicating $50 \%$ digestion by zymolyase and generation of spheroplasts.

\section{Trypan blue staining of cell walls}

Detection of cell walls using Trypan blue was performed as described previously (40). Briefly, $1 \mathrm{ml}$ of log phase $\left(\mathrm{OD}_{600} \sim 0.6-0.8\right)$ culture under aerobic and hypoxic conditions was centrifuged at $10 \mathrm{~K}$ rpm for $2 \mathrm{~min}$. The cells were washed once in PBS and resuspended in $1 \mathrm{ml}$ PBS. Trypan blue was added at a final concentration of $10 \mu \mathrm{g} / \mathrm{ml} .5$ $\mu \mathrm{l}$ of cells were observed on a slide with coverslip using a Leica SP5 confocal microscope. Image processing was performed using Image J. Staining intensity was determined by drawing a region around the stained area, measuring the mean gray value and subtracting the background signal for approximately 300 cells under aerobic conditions and 150 cells under hypoxic conditions.

\section{Chromatin Immunoprecipitation}

Chromatin immunoprecipitation (chIP) was performed as described (41-43). Briefly, cultures were diluted and grown overnight to mid-log phase $\left(\mathrm{OD}_{600} \sim 0.4-0.8\right)$. Cultures 
were then fixed with $1 \%$ formaldehyde for 20 minutes (histone chIPs) or 45 minutes (FLAG-Set4 and Sir3-HA chIPs). For the Rpd3-FLAG chIPs, a double crosslinking strategy was used as previously reported (44) to improve recovery of Rpd3-FLAG with chromatin. In this case, cells were harvested and resuspended in PBS. EGS (ethylene glycol bis (succinimidyl succinate)) was added to a final concentration of $1.5 \mathrm{mM}$ and cells were fixed for $30 \mathrm{~min}$. Then $1 \%$ formaldehyde was added and cells were incubated for an additional $30 \mathrm{~min}$. Quenching was performed with $0.5 \mathrm{M}$ Tris- $\mathrm{HCl} \mathrm{pH} 7.5$ for 10 min. Cells were pelleted and washed 1X with TBS prior to lysis.

Whole cell extracts were made by bead beating and the chromatin was digested with micrococcal nuclease enzyme. The amount of chromatin used was $40 \mu \mathrm{g}$ per IP (histone chIPs) and 100-300 $\mu \mathrm{g}$ per IP (FLAG-Set4, Sir3-HA, and Rpd3-FLAG chIPs). The antibodies were either pre-bound to protein A/G magnetic beads (Pierce) overnight (histone, Sir3-HA, Rpd3-FLAG chIPs) or pre-conjugated anti-FLAG M2 magnetic beads (Sigma-Aldrich) were used (FLAG-Set4 chIPs). The beads were added to the extracts and rotated overnight at $4{ }^{\circ} \mathrm{C}$. Protein-DNA complexes were eluted using $1 \%$ SDS and $0.1 \mathrm{M} \mathrm{NaHCO}_{3}$, cross-links were reversed, and samples were treated with proteinase $\mathrm{K}$ and RNase A. DNA was extracted with phenol-chloroform-isoamyl alcohol and precipitated using ethanol. qPCR was performed as described above using $0.5 \mu \mathrm{l}$ chIP DNA per reaction and gene-specific primers (Table S2). Three technical replicates were performed for each qPCR reaction and a minimum of three biological replicates were performed for each chIP experiment. Percent input was calculated relative to $10 \%$ of the input. The following antibodies were used for chIP: rabbit anti-H4K5ac (AbCam; catalog no. ab51997), rabbit anti-H4K12ac (EMD Millipore; catalog no. ABE532), rabbit antiH4K16ac (EMD Millipore; catalog no. 07-329), rabbit anti-H3 (AbCam; catalog no. ab1791), rabbit anti-H3K4me3 (Active Motif; catalog no. 39159), rabbit anti-H3K9ac (EMD Millipore; catalog no. 06-942), mouse anti-FLAG (Sigma-Aldrich; catalog no. F1804), mouse anti-HA (EMD Millipore; catalog no. 05-904).

\section{$\underline{\text { Results }}$}

\section{Subtelomeric gene expression is disrupted in set4 $\Delta$ mutants}

To better define the contribution of Set4 to gene expression and any potential roles in silent chromatin regulation, we performed an RNA-sequencing experiment on wildtype and set4 $\Delta$ cells in unstressed conditions (mid-log-phase growth, rich medium). Significantly differentially-expressed genes were identified based on $\log _{2}$ fold-change (log FC) in set4 $\Delta$ cells relative to wildtype using local FDR $\leq 0.05$ (see Materials and Methods; Table S3). In this analysis, 196 genes were identified as significantly differentially-expressed in set4 $\Delta$ cells, with 75 genes up-regulated and 121 genes downregulated in the absence of Set4 (Figure 1A, Table 1). We performed gene ontology analysis to identify enriched categories of genes among those differentially-expressed and identified no functional enrichment in genes down-regulated in set4 $\Delta$ cells, though there is enrichment for genes involved in cell wall organization in those up-regulated in set4 $\Delta$ cells (Table $\mathbf{1}$ ). 
We noted that many of the genes associated with cell wall organization are encoded within subtelomeric regions (29). Therefore, we next assessed the enrichment of genes within $40 \mathrm{~kb}$ of chromosome ends to determine whether there is a more general enrichment for differential expression of subtelomeric genes in set4 $\Delta$ cells that is independent of gene functional category. We observed a more than five-fold enrichment in genes adjacent to telomeres $\left(p=1.60 \times 10^{-28}\right.$ for all genes; hypergeometric test; Figure 1B) within those differentially expressed in set4 $\Delta$ mutants. We also analyzed previously published microarray data of set4 $\Delta$ cells grown in synthetic medium (11). Interestingly, the differentially-expressed genes in this dataset also showed significant enrichment for subtelomeric genes ( $p$ value $=0.0003$; Figure 1B), providing further evidence that Set4 may have a specific role in regulating expression of telomere-adjacent genes. In the same dataset (11), gene expression in set3 $\Delta$ cells was also analyzed, which showed no significant enrichment for differential expression of subtelomeric genes ( $p$ value $=0.115$ ). Together, these data suggest that under normal growth conditions, Set4 plays a specific role in regulating telomere-adjacent genes and genes linked to cell wall organization.

To further investigate these findings, we performed targeted gene expression analysis of wildtype and set4 $\Delta$ cells using RT-qPCR. We investigated two classes of subtelomeric genes: (1) one set of genes (COS12, YGL262W, and YPS5) on the left arm of chromosome seven adjacent to TELO7L, a well-characterized site for chromatinbased silencing and telomere position effect; and (2), the seripauperin (PAU) genes, a highly homologous, subtelomeric gene family induced during different stresses-particularly anaerobic growth-that are thought to be important for cell wall remodeling or sterol uptake during stress (Figure 1C) $(45,46)$. We monitored expression of PAU11, which is also located adjacent to TELO7L, and PAU13, using primers that uniquely amplify these genes (Table S2), as well as PAU21 and PAU22, which have identical sequences (indicated as PAU21/22 where appropriate). In the absence of Set4, COS12, YGL262W and YPS5 were downregulated, whereas PAU11, PAU13, and PAU21/22 were upregulated. These data suggest Set4 is important for both maintaining expression of some subtelomeric genes and repressing other subtelomeric genes under physiological, unstressed conditions.

The pattern of neighboring gene expression changes observed in set4 $\Delta$ cells is consistent with a role for Set4 in altering regional chromatin structure. We therefore tested whether set4 $\Delta$ cells showed any defects in a canonical telomere position effect (TPE) assay using a strain carrying URA3 integrated near TELO7L. In this reporter assay, we did not observe any substantial change in URA3 expression in the absence of Set4 or Set3, unlike the loss of silencing observed in set1 1 cells (Figure S2A). We also analyzed telomere length by Southern blot, which showed no difference between wildtype and set4 $\Delta$ cells in the length of terminal telomere restriction fragments (Figure S2B). This indicates that Set4 has a specific regulatory role distinct from other TPE regulators and is not required for telomere length maintenance under normal conditions.

\section{Deregulation of gene expression is enhanced in set $4 \Delta$ mutants during stress}


In previous work, we demonstrated that Set4 promotes proper gene expression in response to oxidative stress (10). Many genes encoded within subtelomeres are stress response genes; therefore we analyzed whether some of these genes showed Set4-dependent changes in expression during oxidative stress. Upon treatment with hydrogen peroxide, genes that were downregulated in set4 $\Delta$ cells did not show substantial change (COS12 and YGL262W; Figure S3A). Genes that were upregulated in set4A cells under normal conditions (e.g. PAU13 and PAU21/22) were more highly upregulated in set4 $\Delta$ cells in the presence of hydrogen peroxide, although repressed in wildtype cells, indicating that the loss of Set4 attenuates their repression in hydrogen peroxide.

Serratore et al. (14) previously showed that Set4 is important for the regulation of gene expression in hypoxic conditions and that hypoxia causes an increase in Set4 protein levels. As the PAU genes are highly upregulated during hypoxia (46), we tested their expression in set4 $\Delta$ cells, along with the other telomere genes, under hypoxic conditions. We first tested our growth conditions for wildtype cells grown in aerobic and hypoxic conditions. We observed that we obtained the most consistent results by diluting stationary phase cultures to very low $\mathrm{OD}_{600}$ and allowing them to grow to $\mathrm{OD}_{600}$ 0.4-0.8 over the course of 18 hours in hypoxia, similar to how we tested set4 $\Delta$ mutants sensitivity to oxidative stress $(10,12)$. Under these conditions, the PAU genes were highly upregulated, and there was also significant upregulation of $Y G L 262 W$, although expression of COS12 and YPS5 at TELO7L remained mostly unchanged in hypoxic compared to aerobic growth (Figure 2A). In the set4 $\Delta$ strain grown under hypoxia, COS12 and YGL262W expression showed no or minimal decrease in hypoxic conditions, whereas the expression of PAU11, PAU13, PAU21/22, and YPS5 was significantly increased over the level of induction seen in wildtype cells (Figure 2B). These data indicate that the loss of Set4 leads to enhanced induction of hypoxiaregulated genes, including genes that are both negatively- and positively-regulated by Set4 under aerobic conditions (e.g., the PAU genes and YPS5, respectively). These observations parallel our findings in hydrogen peroxide treated cells, in which repression is inhibited at PAU13, PAU21/22, and YPS5 (Figure S3A), indicative of a common gene regulatory role for Set4 under different stress conditions.

To address the role of Set4 in regulating subtelomeric genes more broadly during stress, we performed RNA-sequencing of wildtype and set4 $\Delta$ cells grown under hypoxic conditions. In wildtype cells, growth in hypoxia induced widespread gene expression changes with 1056 genes up-regulated and 835 genes down-regulated ( $\log \mathrm{FC} \geq 1.0, p$ $\leq$ 0.05; Table S3, Figure 2C). The significantly differentially-expressed genes encompassed a range of GO categories, including enrichment for genes associated with transmembrane transport, lipid metabolic process, and cell wall organization, among others, in the up-regulated genes (Table S4). The genes down-regulated in wildtype cells in hypoxia were highly-enriched for translation associated processes, mitotic cell cycle, cytoskeletal organization, cell wall organization, and lipid metabolic processes, among others (Table S4). The gene expression changes reported here are similar to those previously-described under hypoxic or anerobic growth of yeast $(47,48)$. 
In set4 $\Delta$ cells, we observed a largely similar cohort of differentially expressed genes in hypoxia as in wildtype cells, with 1073 genes up-regulated and 917 genes down-regulated ( $\log F C \geq 1.0, p \leq 0.05$; Table S3, Figure 2D). These genes encompassed similar GO categories to those observed in wildtype cells (Table S4). There are more genes down-regulated in set $4 \Delta$ cells grown in hypoxia compared to the total number of genes down-regulated in wildtype cells. These genes are distributed across a number of functional categories, including GO terms associated with translation-related processes, cytoskeletal organization, and DNA repair (Table S4).

When directly comparing wildtype and set4 $\Delta$ cells in hypoxia, we identified 377 total genes differentially-expressed, with 205 genes up-regulated and 172 genes downregulated in the absence of Set4 (Table 1; Figure 2E). Gene ontology analysis revealed enrichment for genes associated with cell wall organization in both the up- and down-regulated sets of genes and genes linked to DNA integration also enriched in the down-regulated genes (Table 1). Compared to aerobic conditions, this represents an increased number of cell wall organization genes misregulated in the absence of Set4. Interestingly, the down-regulated genes associated with the GO term DNA integration are almost entirely from Ty transposable elements (Table S3). Given that these are not differentially-expressed under aerobic conditions, this indicates enhanced repression of these genes without Set4 under hypoxia. In addition, previous work showed differential regulation of ergosterol biosynthetic genes in set4 $\Delta$ mutants grown under hypoxia (14). However, we did not observe enrichment of ergosterol biosynthetic genes within the differentially-expressed gene set from our RNA-sequencing experiments (Table S3), nor by directly testing ERG3 and ERG11 expression using RT-qPCR (Fig. S3B). It is possible that differences in yeast strains or growth conditions, such as the time in hypoxia, may contribute to this difference in expression patterns.

Based on results obtained under aerobic conditions and RT-qPCR experiments performed on telomere genes, we predicted that genes with altered expression in set4 $\Delta$ cells in hypoxia may show subtelomeric enrichment. Indeed, for those genes upregulated in hypoxic set4 $\Delta$ cells, there was six-fold enrichment for subtelomeric localization compared to expected ( $p=2.79 \times 10^{-31}$; hypergeometric test; Figure 2F) and almost two-fold enrichment for subtelomeric localization for down-regulated genes $(p=0.008)$. These data support our conclusions from the RT-qPCR experiments indicating enhanced expression changes in cell wall organization genes at subtelomeres in set4A cells under hypoxia and indicate a broad role for Set4 in regulating subtelomeric genes genome-wide under both normal and stress conditions.

\section{Set4 maintains cell wall integrity during hypoxic growth}

Our previous work identified a role for Set4 in protecting cells during oxidative stress, likely through the regulation of gene expression. We showed that loss of Set4 increases sensitivity to oxidizing agents such as hydrogen peroxide, and Set4 overexpression increases survival upon hydrogen peroxide treatment (10). When growing cells under hypoxic conditions, we observed that set4 $\Delta$ mutants grew more 
slowly and had smaller colony sizes (Figure 3A), indicating impaired growth under hypoxia. We hypothesized that the deregulated expression of the PAU genes, as well as other hypoxia-induced genes, may lead to disrupted cell wall integrity. We assayed cell growth in hypoxia with sorbitol to increase the osmolarity and suppress cell wall defects. As shown in Figure 3A, the growth differential between wildtype and set4A cells was decreased in the presence of sorbitol, suggesting stabilization of any cell wall defects in these cells. In addition, we tested the sensitivity of our strains to zymolyase digestion, which targets $\beta-1,3$ glucan linkages in the cell wall. As previously-shown (49), yeast grown under hypoxic conditions showed increased resistance to zymolyase compared to aerobic conditions (Figure 3B). However, set4A cells showed modestly more sensitivity to zymolyase digestion than wildtype cells in hypoxia, further indicating disrupted cell wall integrity.

Yeast cell walls show altered thickness and composition in hypoxia (49), which can be visualized using trypan blue, which stains yeast glucans and chitin (40). In set4A cells under aerobic conditions, we observed increased trypan blue staining of cell walls compared to wildtype (Figure $3 \mathbf{C}$ ). In wildtype cells in hypoxia, chitin composition and cell wall mass decrease, lowering trypan blue staining (40). We observed an expected decrease in wildtype cells grown in hypoxia (Figure $3 \mathrm{C}$ ), although staining remained relatively high in set4 $\Delta$ cells in hypoxia, indicating an attenuation of this component of the hypoxic response. Altogether, these data are consistent with our previous findings of altered stress responses in the absence of Set4 (10).

\section{Set4 maintains histone acetylation levels at stress response genes within subtelomeric regions}

Our data show deregulation of hypoxia response genes in the absence of Set4, particularly those located within subtelomeric regions and important for cell wall integrity. Multiple histone deacetylases (HDACs) have been shown to control expression of stress response genes within subtelomeric regions and are key regulators of the repressive chromatin environment at subtelomeres (26-29). Orthologs of Set4 in other organisms and the yeast protein Set3 are known to interact with or otherwise regulate the activity of HDACs in different chromatin environments $(7,9,19,21,50,51)$. Thus, we hypothesized that Set4 may play a similar role at subtelomeric regions in yeast. To investigate this further, we tested the distribution of a series of acetylation marks previously implicated in the regulation of subtelomeric chromatin, including $\mathrm{H} 4 \mathrm{~K} 5 \mathrm{ac}, \mathrm{H} 4 \mathrm{~K} 12 \mathrm{ac}, \mathrm{H} 4 \mathrm{~K} 16 \mathrm{ac}, \mathrm{H} 3 \mathrm{~K} 9 \mathrm{ac}$, as well as the transcription-associated mark H3K4me3. We tested for association of Set4 at telomeric regions (TELO7L), as well as an internal site $12 \mathrm{~kb}$ from the chromosome end which marks the approximate boundary with euchromatin (TELO7L $L_{\text {boundary }}$ ). We also tested localization at the promoters of the $P A U$ genes in wildtype and set4 $\Delta$ cells. In aerobic conditions, we observed lower levels of histone acetylation close to the telomere (TELOTL primer set), and increased acetylation levels and $\mathrm{H} 3 \mathrm{~K} 4 \mathrm{me} 3$ at more distal regions such as the TELO7L boundary region (Figure 4A). This is the expected distribution pattern of histone acetylation and H3K4me3 at subtelomeres and provides both positive and negative controls for the chIP. 
In the absence of Set4, there was relatively little change in the abundance of these marks at any of the regions tested under aerobic growth. However, in hypoxic conditions, we observed increased acetylation, particularly at telomere-distal locations and the promoters of the PAU genes (Figure 4B). The largest increase in acetylation was observed for H3K9ac, although H4K16ac and H4K5ac also showed marked increases at subtelomeric regions in set4 $\Delta$ cells. The overall abundance of histone acetyl marks was not changed in set4 $\Delta$ cells, although we did observe a global decrease in H4K16ac in hypoxic conditions compared to aerobic conditions (Figure S4A). We also note that there was no change in H3K4me3 levels in set4A cells in aerobic or hypoxic conditions (Figure 4A-4B), indicating that this mark is not regulated by Set4 at subtelomeric regions. Combined, these findings demonstrate increased acetylation at multiple histone sites upon loss of Set4 in hypoxic conditions, consistent with our observations of enhanced activation of the PAU genes and less repression of other subtelomeric genes (e.g. COS12, YGL262W) in set4 $\Delta$ cells.

\section{Disrupted localization of HDACs at subtelomeric regions in set4A mutants}

The HDACs Sir2 and Rpd3 are both known regulators of silent chromatin near telomeres $(17,18,27,52)$ and have also been implicated in the regulation of stress response genes, including those induced during hypoxic or anaerobic growth $(26,28,29,53)$. These observations, and our findings of altered levels of histone acetylation in the absence of Set4, led us to test the hypothesis that Set4 works with HDACs to maintain telomeric chromatin structure. We investigated the distribution of Rpd3 and the SIR complex in wildtype and set4A cells under hypoxic conditions. We focused on hypoxia as both the gene expression data and histone modification chIP data suggest a much larger dependence on Set4 in hypoxic than aerobic conditions.

Chromatin immunoprecipitation of epitope-tagged Sir3, which is the direct chromatin-binding component of the SIR protein complex, was performed in set $4 \Delta$ cells under hypoxic conditions. We observed the expected occupancy of Sir3-HA primarily near telomeric chromatin at TELO7L, as well as secondary localization at the promoters of PAU genes, as previously demonstrated in aerobic conditions (28). In hypoxia, Sir3$\mathrm{HA}$ localization at telomeric chromatin decreased in set4 $\Delta$ cells relative to wildtype (Figure 5A), suggesting that Set4 promotes the proper association of the SIR complex with telomeres in these conditions. In agreement with previous findings (28), we observed more binding of Sir3 to PAU13 and PAU11 promoters compared to PAU21/22 promoters, indicating that PAU13 and PAU11 may be more dependent on the SIR complex for maintaining repression. These findings are also consistent with the increase in acetylation in the region observed in hypoxia (Figure 4), including H4K16ac, the primary substrate of Sir2. While we did not observe any differences in protein expression levels of Sir3-HA between wildtype and set4 4 cells, nor between aerobic and hypoxic conditions (Figure S4B), the increased chromatin association of Sir3 in hypoxia in wildtype cells (Figure S5A), which is consistent with the global decrease in H4K16ac levels observed in hypoxia-treated cells (Figure S4A). 
We similarly evaluated the distribution of Rpd3-FLAG at subtelomeres in the absence of Set4. In hypoxic conditions, Rpd3-FLAG showed decreased binding in the absence of Set4 (Figure 5B) indicating that Set4 promotes the localization of Rpd3FLAG to subtelomeric regions. There were no changes in total Rpd3-FLAG protein levels in set4 $\Delta$ mutants (Figure S4C). These observations are consistent with increased acetylation within the region in set4 $\Delta$ cells (Figure 4) and the altered gene expression patterns observed in set $4 \Delta$ cells.

To further define the interaction of Set4 with the Sir2 and Rpd3 HDACs in regulating gene expression, we generated double mutant strains carrying set4 $\Delta$ and rpd3A or sir2 $\Delta$ and monitored gene expression changes and cell growth in hypoxia. Both set $4 \Delta$ and rpd3 $\Delta$ cells showed growth defects in hypoxic conditions compared to aerobic conditions, although there was no clear defect in sir2 $\Delta$ cells and sir2 $\triangle$ set4 $\Delta$ cells grew very similarly to set4 $\Delta$ single mutants (Figure S5B). The loss of Rpd3 resulted in a severe growth defect in hypoxia (Figure S5C), and rpd3 $\Delta$ set4 $\Delta$ cells grew similarly to rpd3 $\Delta$ single mutants, suggesting that Rpd3 and Set4 may contribute to a shared pathway regulating growth in hypoxia.

We next evaluated gene expression at subtelomeres in these single and double mutant strains. As expected, we observed de-repression of telomere-adjacent genes in sir2 $\Delta$ cells under aerobic conditions (Figure S5D). In contrast, we observed enhanced repression of telomere-adjacent genes COS12, YGL262W, and YPS5, as well as lower expression of two out of three PAU genes tested, PAU11 and PAU13, in rpd3A cells (Figure S5D). These data are consistent with previous reports indicating an antisilencing function for Rpd3 at subtelomeres (27).

In sir24 cells grown in hypoxic conditions, COS12 and YGL262W were derepressed, as expected (Figure 6A). Similar expression was observed in the sir2 $\triangle$ set4 $\Delta$ mutants, indicating that expression levels of these genes are largely regulated by the SIR complex in hypoxia. However, we observed a different expression pattern of genes that show enhanced activation in set4 $\Delta$ cells in hypoxia, including YPS5 and the $P A U$ genes. These genes showed increased repression in sir2 $\Delta$ cells compared to wildtype in hypoxia, however this repression was alleviated in the sir2 $\Delta$ set4 $\Delta$ double mutants. PAU11 and PAU13 were expressed at a similar level in the double mutant as wildtype cells, whereas PAU21/22 and YPS5 showed slightly higher expression than in wildtype. These data indicate an antagonistic function of the SIR complex and Set4 in balancing the expression of hypoxia-induced genes.

We also investigated changes in gene expression in the rpd3 $\Delta$ set4 $\Delta$ strain under hypoxic conditions. Rpd3 has been directly implicated in regulating the expression of genes induced during anaerobic growth (26). In the absence of Rpd3, repression of all of the subtelomeric genes remained largely intact compared to the induction observed in wildtype and set4 $\Delta$ cells in hypoxic conditions. However, in the rpd3 $\Delta$ set $4 \Delta$ cells, repression of the $P A U$ genes was relieved and induction closer to wildtype expression levels was observed (Figure 6B). The telomere-adjacent genes COS12 and YGL262W 
showed similar expression levels in rpd3 $\Delta$ and rpd3 $\Delta$ set4 $\Delta$ cells, indicating that loss of Set4 was not sufficient to overcome repression of these genes in the absence of Rpd3. These data suggest that, similar to its interaction with the SIR complex, Set4 counterbalances Rpd3 function in regulating expression of the PAU genes (and likely other genes induced in limiting oxygen). However, this is a gene-specific interaction, as Set4 and Rpd3 appear to function independently at other telomere adjacent genes such as COS12, YGL262W and YPS5.

\section{Set4 localizes to subtelomeric chromatin in hypoxia}

Previous work from our lab showed that Set4 is a chromatin-associated protein and localizes to the promoters of genes that are induced during oxidative stress, particularly in the presence of stress (10). Another report has also shown that Set4 localizes to promoters of ergosterol biosynthetic genes during hypoxia (14). To investigate the localization of Set4 at subtelomeres and whether the changes in subtelomeric gene expression and acetylation levels are due to local occupancy by Set4, we performed chromatin immunoprecipitation (chIP) under both aerobic and hypoxic conditions using a strain expressing N-terminally FLAG-tagged Set4 from its endogenous locus and monitored binding to TELO7L and TELO7L $L_{\text {boundary }}$ regions and at the promoters of PAU genes. In aerobic conditions, we did not detect significant association of Set4 at any of these regions (Figure S6). However, under hypoxia, we observed binding of Set4 to a region near TELO7L as well as the promoters of the PAU genes (Figure 7). This binding was enhanced relative to a negative control region at CENVX. In addition, we also tested the promoters of other non-telomeric genes known to be regulated by Set4 during stress (CTT1, PNC1, ERG3, and ERG11) $(10,14)$. Set4 localized to the promoters of CTT1 and PNC1, as expected based on our previous findings (10), and was highly enriched at ERG3 and ERG11 gene promoter (Figure 7). These results are consistent with a previous report showing binding to ERG3 and ERG11 promoters in hypoxia (14), however our data indicate that these are not a major site for gene regulation by Set4 under similar conditions (Figure S3B).

The binding of Set4 to subtelomeric chromatin under stress suggests that Set4 may directly influence gene expression within the region. Set4 expression increases dramatically during hypoxia (14), therefore we expect increased association with chromatin under these conditions. In aerobic conditions, the abundance of Set4 is very low (10), which likely limits our ability to detect it by chIP. Combined with our gene expression data, we expect that Set4 may be present at subtelomeric chromatin at levels below the limit of detection in aerobic conditions, and Set4 abundance and localization near telomeres increases in hypoxia.

\section{Discussion}

Yeast subtelomeres are enriched for stress response genes, and proteins orthologous to Set4 are known regulators of heterochromatin and gene silencing (1922). Previous studies have highlighted a role for Set4 as a calibrator of stress- 
responsive gene expression $(10,14)$. Here, we uncovered a role for Set4 in regulating genes within the repressed subtelomeric regions of budding yeast under both normal and stress conditions, particularly during hypoxia. Gene expression and chromatin immunoprecipitation analysis indicate that Set4 works together with the SIR complex and Rpd3 within the subtelomeres to fine-tune expression levels of stress-response genes. The loss of Set4 also decreases survival and cell wall integrity in hypoxia. Therefore, Set4 helps to maintain the proper balance of expression of stress response genes to promote survival during stress.

\section{Set4-dependent regulation of subtelomeric gene expression under both normal and stress conditions}

Previous work has shown that Set4 localizes to the promoters of oxidative stressinduced genes following hydrogen peroxide treatment (10) and ergosterol biosynthetic genes during hypoxia (14). Set4 is lowly expressed under normal conditions, and its localization to these promoters was only detected during stress. We also observed enrichment of Set4 within subtelomeric regions, specifically during hypoxia, when Set 4 protein abundance is dramatically increased (14). Changes in gene expression of telomere-adjacent genes and the stress-induced PAU genes were observed under both normal and stress conditions; however, the dependence on Set4 was clearly enhanced during stress. RNA-seq revealed an overall increase in differential gene expression between wildtype and set4 $\Delta$ cells in hypoxia, although we note that there was little change in the pattern of gene expression changes observed compared to aerobic conditions (GO analysis and genomic location).

Consistent with changes in gene expression, there were greater changes in histone acetylation levels in hypoxia compared to normal conditions in set $4 \Delta$ cells. We postulate that Set4 is present within subtelomeres (and likely other chromatin regions) even under normal, unstressed conditions, as we observe Set4-dependent changes in gene expression; however, the standard chIP assay is not sufficiently sensitive to detect this low abundance protein. In hypoxic conditions, the differences in gene expression and histone acetylation in set $4 \Delta$ cells compared to wildtype cells are exacerbated, and we observe a clear localization of Set4 to subtelomeric chromatin. The increased abundance of Set4 in hypoxia (14) allows us to readily detect the protein using chIP. Combined with our previous results showing increased chromatin association of Set4 during oxidative stress (10), these data indicate that the gene regulatory role for Set 4 is more critical during stress. This suggests that one component of the cellular response to certain types of stress is to increase Set4 protein levels and/or increase its association with chromatin to promote stress-responsive gene expression programs. Currently, this role for Set4 has only been linked to oxidative stress and limiting oxygen (hypoxic or anaerobic) conditions. It remains to be determined whether or not Set4 is a general stress response factor, similar to the Msn2 and Msn4 transcription factors (54), or if it has a specialized role under certain types of stress. 


\section{Set4 coordinates histone deacetylases to regulate subtelomeric chromatin structure}

The chromatin structure at subtelomeric regions of $S$. cerevisiae is maintained by multiple HDACs to generate a hypoacetylated state, which keeps gene expression levels low $(17,18)$. Members of the Set3 subfamily of SET-domain proteins, including Set3, UpSET and SETD5 are all known to physically interact with histone deacetylases (7), and loss of function of these proteins leads to aberrantly high levels of histone acetylation $(8,21,51)$. Protein-protein interaction analysis under hypoxic conditions revealed interactions of Set4 with other chromatin regulators, though not HDAC complex members (14), however further analysis may reveal how Set4 influences HDAC function. Using chromatin immunoprecipitation, we observed decreased binding of both the SIR complex and Rpd3 within subtelomeric regions in cells lacking Set4 under hypoxic conditions, when Set4 expression is high. Not all Rpd3 or SIR protein binding is lost in the absence of Set4, suggesting other targeting mechanisms of both HDACs are still intact. However, the reduced presence of each of these HDACs is consistent with increases in local histone acetylation in set4 $\Delta$ cells in hypoxia (Figure 8). In addition, gene expression analysis in sir2 $\Delta$ set $4 \Delta$ and rpd3 $\Delta$ set $4 \Delta$ double mutants indicated that the repression of the PAU genes observed in the absence of either HDAC alone is relieved upon loss of Set4. At subtelomeric chromatin, Rpd3 has been reported to antagonize the spread of the SIR complex in silent chromatin regions $(17,27,52)$. Our data suggests a model in which the loss of Set4 diminishes the ability of either Sir2 or Rpd3 to fine-tune PAU gene expression levels, causing aberrantly high activation of these genes. Despite the antagonism between the SIR complex and Rpd3, the reduction in both of their levels in set4 $\Delta$ mutants likely reduces aberrant spreading of the HDACs, allowing PAU gene expression to approach or exceed wildtype levels in set4 $\Delta$ sir2 $\Delta$ and set4 $\Delta$ rpd3 $\Delta$ mutants.

It is also feasible that, in addition to maintaining proper SIR complex and Rpd3 levels at subtelomeres, Set4 works alone or in cooperation with these HDACs to inhibit association of a positive regulator of hypoxia-induced genes. Previous work has demonstrated that Rpd3 promotes the association of the transcription factor Upc2 with some anaerobic response genes (26). Upc2 is required for the induction of the PAU genes and works with the SAGA transcriptional activator and histone acetyltransferase complex to promote PAU gene expression in hypoxia (55). Interestingly, it has been reported that Set4 blocks Upc2 activity at ergosterol biosynthetic genes, thereby repressing these genes in hypoxia (14). Determining the interaction of Set4 with Upc2 and Rpd3 at the PAU genes may shed further light on regulatory mechanisms controlling their expression in hypoxia.

Altogether, this study provides new insights into the types of genes regulated by Set4 and the chromatin-based mechanisms through which it acts, as well as identifies a new telomere regulator in stress conditions. We have identified a role for Set4 in maintaining heterochromatic structures in yeast, which aligns its functions with metazoan orthologs previously implicated in heterochromatin maintenance (20-22), and expands our understanding of the role for Set4 during stress. Our data indicating 
decreased fitness and cell wall integrity of cells lacking Set4 in hypoxic conditions support the conclusion that Set4 promotes cell survival during stress, which is consistent with our previous findings identifying a role for Set 4 in protecting cells during oxidative stress (10). Additional studies of Set4, and other Set3-related proteins, are likely to further our understanding of gene regulatory mechanisms and chromatinmediated stress defense pathways. 


\section{Data availability}

The raw and processed data for RNA-sequencing experiments is available on the Gene Expression Omnibus database at accession number GSE173901.

\section{Funding}

This work was support by the National Institutes of Health [R01GM124342 to EMG] and the National Research Foundation of Korea [NRF-2019H1D3A2A02102167 to DP].

\section{Acknowledgements}

The authors thank all members of the Green lab for helpful discussions, technical assistance, and feedback on the manuscript. We thank Dr. Paul Kaufman for yeast strains and Dr. Philip Farabaugh for sharing equipment. We are also grateful to Tagide deCarvalho of the Keith R. Porter Imaging Facility for assistance with microscopy. 
Table 1. Significant differentially-expressed genes in set4A mutants compared to wildtype under aerobic and hypoxic conditions.

\begin{tabular}{|c|c|c|c|}
\hline & Total genes & Up-regulated & Down-regulated \\
\hline Aerobic & 196 & 75 & $\begin{array}{l}121 \\
\text { No enrichment }\end{array}$ \\
\hline Hypoxic & 377 & $\begin{array}{l}205 \\
\text { Cell wall organization }\left(3 \times 10^{-11}\right)\end{array}$ & $\begin{array}{l}172 \\
\text { Cell wall organization }\left(2 \times 10^{-04}\right) \\
\text { DNA Integration }\left(2 \times 10^{-05}\right)\end{array}$ \\
\hline
\end{tabular}

The number of significant differentially expressed genes in each category is indicated along with the enriched GO terms with $p$ values indicated in parentheses. 
Figure 1

A

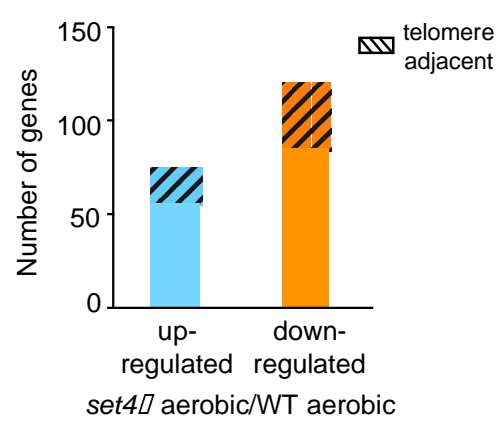

C
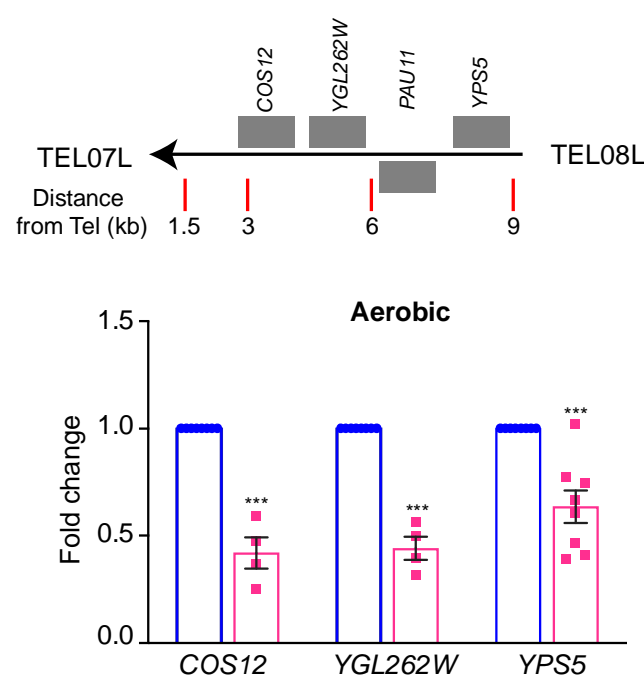

B

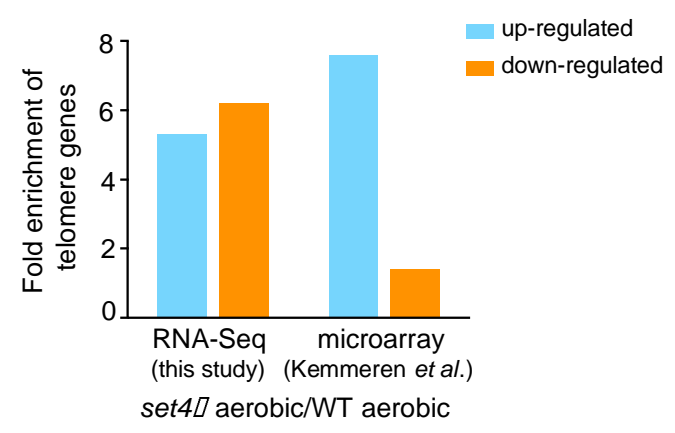

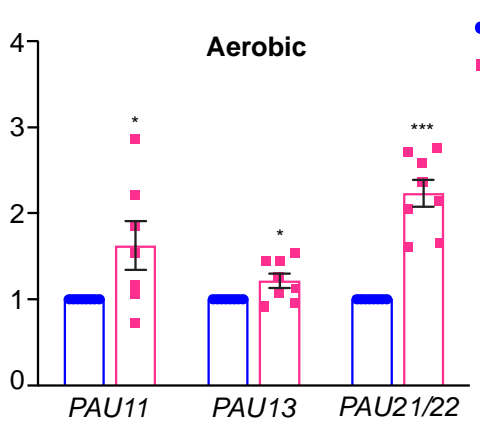

Figure 1: Set4 regulates the expression of subtelomeric genes.

A. The total number of genes identified as up- or down-regulated (FDR $<0.05)$ from RNA-sequencing of set4A (yEG322) cells relative to wt (yEG001). Gene list provided in Table S3. The total number of telomere-enriched genes are indicated with the hashed box. B. The fold enrichment of differentially expressed subtelomeric genes (defined as less than $40 \mathrm{~kb}$ from the chromosome end) in our RNA-sequencing data of set4 $\Delta$ cells and in previously published microarray data (11). C. RT-qPCR of sub-telomeric genes from wt (yEG001) and set4A (yEG322) strains grown in YPD. Expression levels were normalized to TFC1. Fold change relative to wt is shown. The error bars represent S.E.M. from at least three biological replicates. Asterisks represent $p$ values as calculated by an unpaired $t$ test $\left({ }^{*}<0.05,{ }^{* *}<0.01,{ }^{* * *}<0.001\right)$. 
Figure 2

A

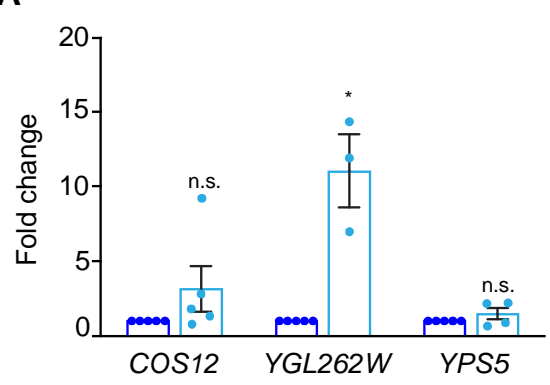

B

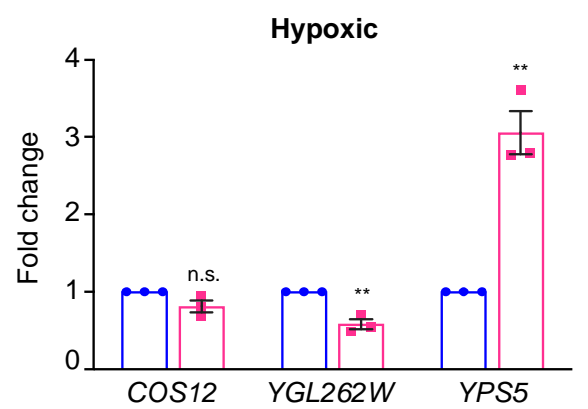

C

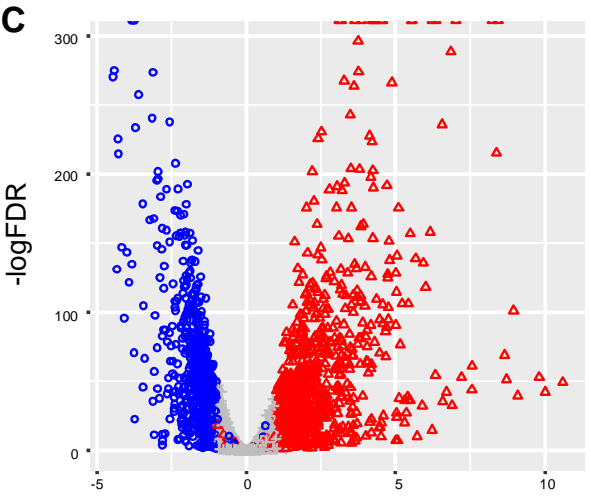

Log $_{2}$ fold-change (WT hypoxic/WT aerobic)

$\mathbf{E}$

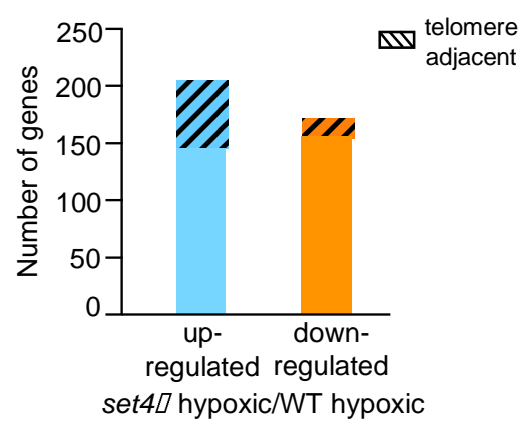

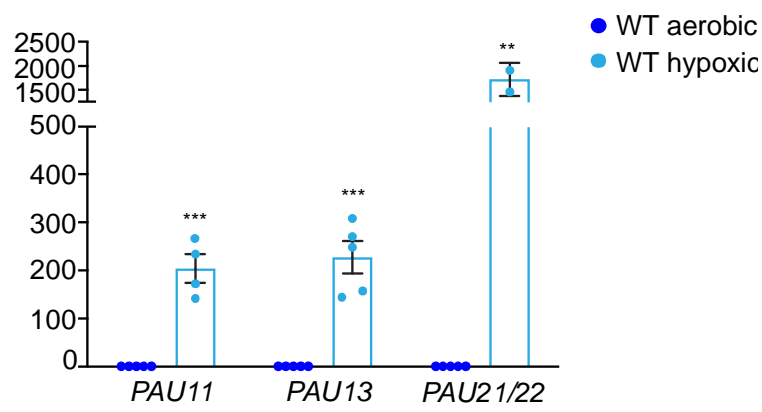
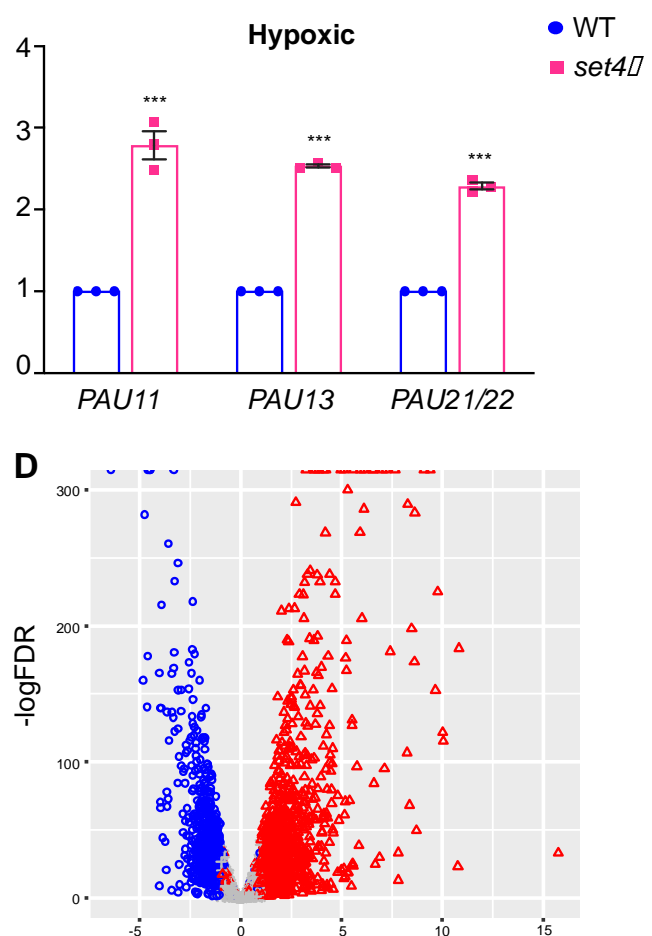

$\log _{2}$ fold-change (set $4 \square$ hypoxic/set $4 \square$ aerobic)

$\mathbf{F}$

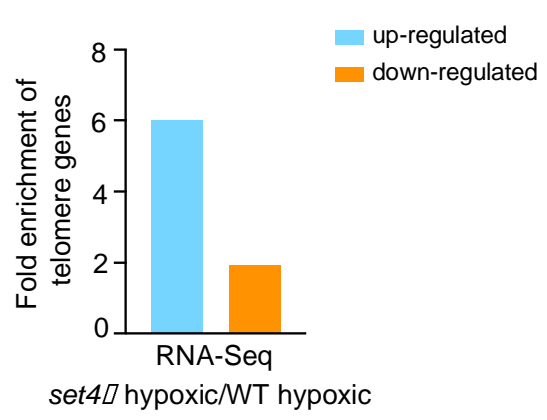

Figure 2: Stress response genes at subtelomeres are regulated by Set4. 
A. RT-qPCR of sub-telomeric genes from wt (yEG001) strains grown in YPD under aerobic or hypoxic conditions. Expression levels were normalized to TFC1 and fold change relative to aerobic conditions is shown. B. RT-qPCR of subtelomeric genes from wt (yEG001) and set4A (yEG322) strains grown in hypoxia in YPD. Expression levels were normalized to TFC1. Fold change relative to wt in hypoxia is shown. For all panels, error bars represent S.E.M. from at least three biological replicates and asterisks represent $p$ values as calculated by an unpaired t test $\left({ }^{*}<0.05,{ }^{* *}<0.01,{ }^{* * *}<0.001\right)$. C, D. Volcano plots depicting significantly differentially expressed genes ( $\log F C \geq 1.0, p \leq$ $0.05)$ comparing wildtype hypoxic to wildtype aerobic cultures (C) and set4 $\Delta$ hypoxic to set4 $\Delta$ aerobic cultures (D). E. The total number of genes identified as up- or downregulated (FDR < 0.05) from RNA-sequencing of set4A (yEG322) cells relative to wt (yEG001) in hypoxia. Gene list provided in Table S3. The total number of telomereenriched genes are indicated with the hashed box. F. The fold enrichment of differentially expressed subtelomeric genes (defined as less than $40 \mathrm{~kb}$ from the chromosome end) for those genes differentially-expressed between wildtype hypoxic cultures and set $4 \Delta$ hypoxic cultures. 
Figure 3

A

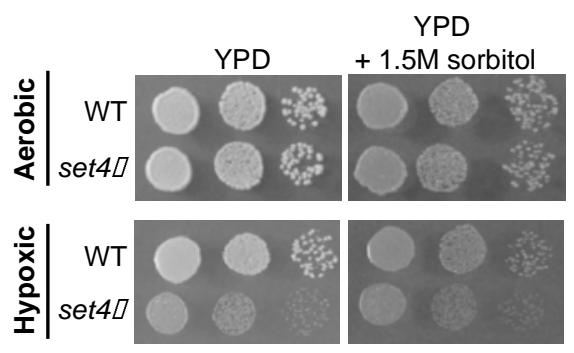

C

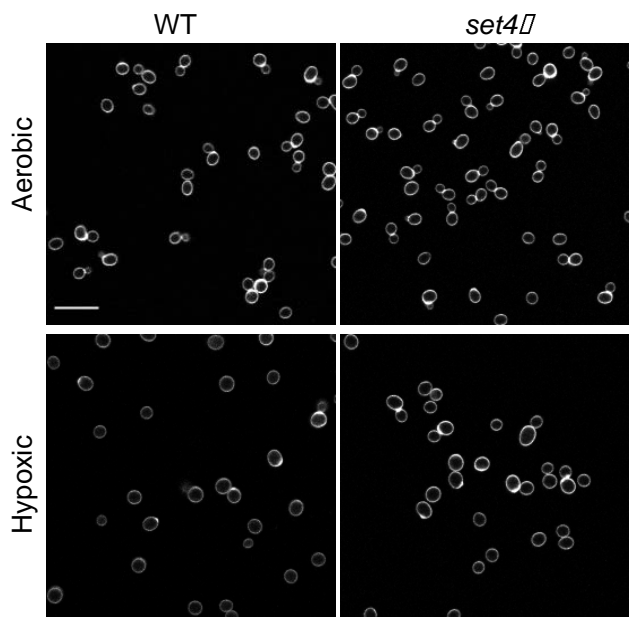

B
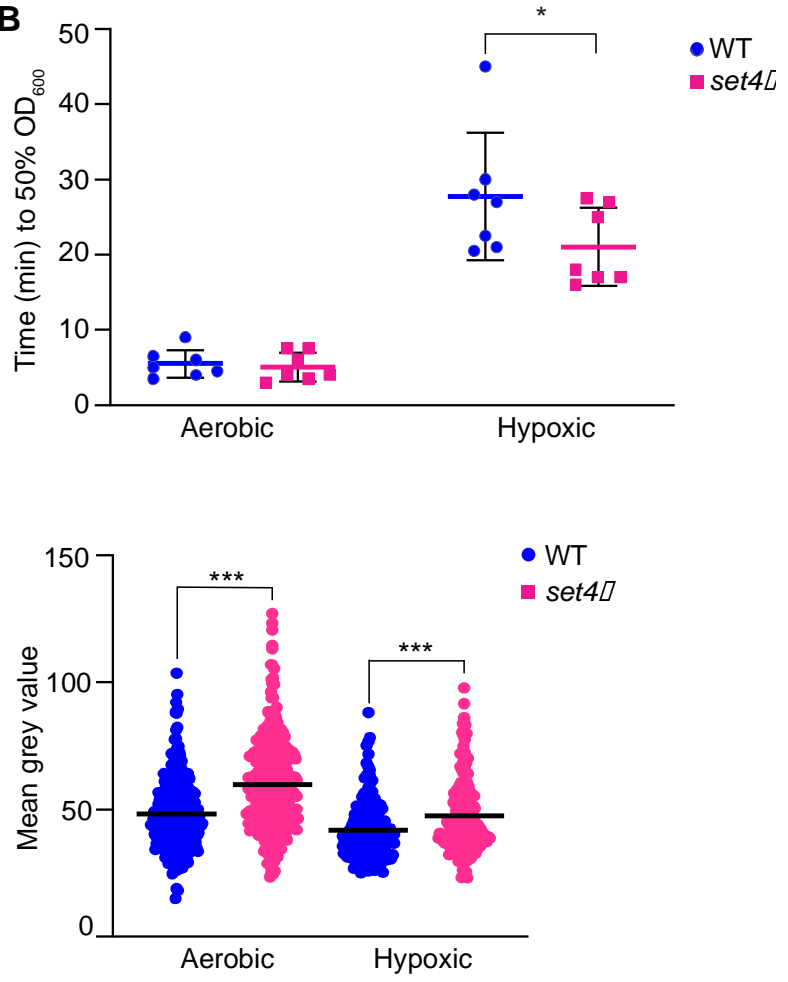

Figure 3: Set4 promotes cell fitness and cell wall integrity in hypoxia.

A. Ten-fold serial dilutions of wt (yEG001) and set4A (yEG322) strains spotted on YPD or YPD with sorbitol and grown under aerobic (2 days) or hypoxic (8 days) conditions at $30^{\circ} \mathrm{C}$. B. Scatter dot plot of the time elapsed for wt (yEG001) and set4 $\Delta$ (yEG322) cultures grown in either aerobic or hypoxic conditions to reach $50 \%$ digestion by zymolyase. Error bars represent standard deviation (S.D.) from seven biological replicates. Asterisk represents $p$ value as calculated by two-way ANOVA and Sidak's multiple comparisons test $\left({ }^{*}<0.05\right)$. C. Fluorescence microscopy of wt (yEG001) and set4A (yEG322) cells grown under aerobic or hypoxic conditions and stained with Trypan blue. Quantitation of the mean gray value for approximately 300 cells under aerobic conditions and 150 cells under hypoxic conditions. Asterisk represents $p$ value as calculated from an unpaired $t$ test $\left({ }^{* *}<0.001\right)$. 


\section{Figure 4}

A
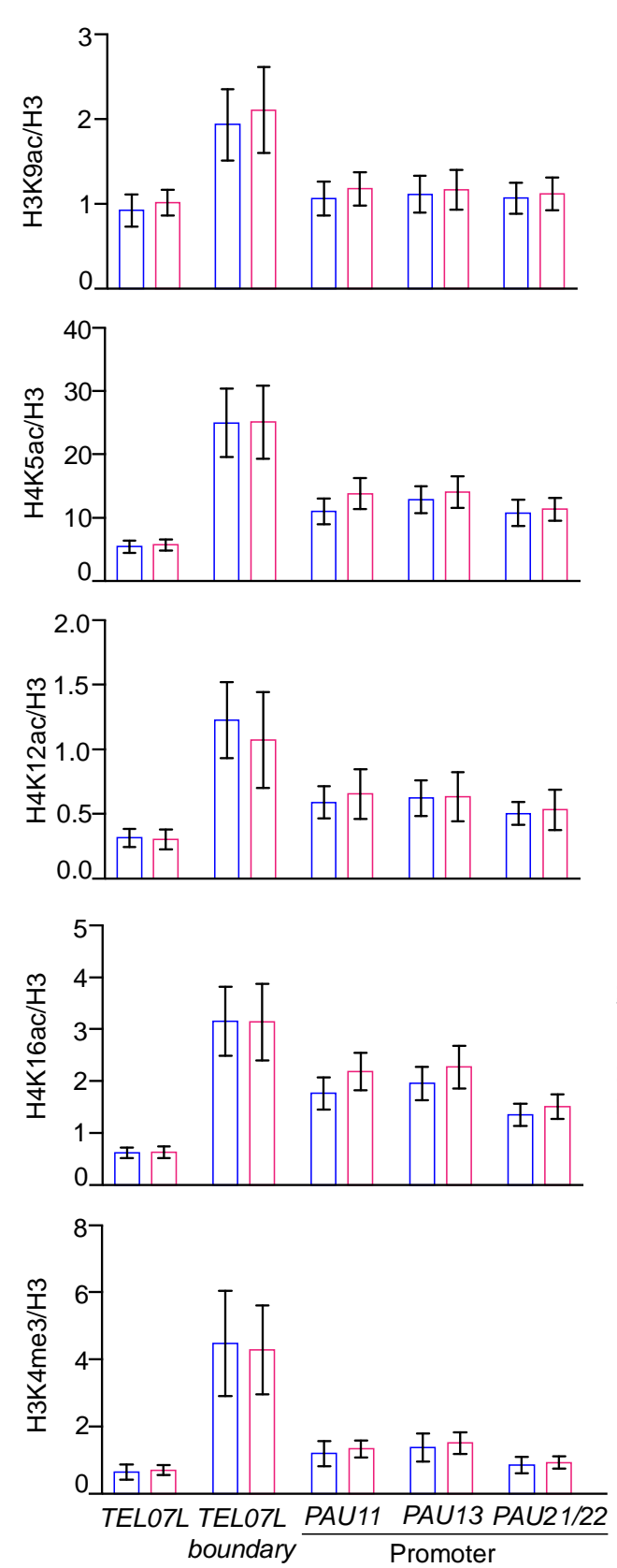

B
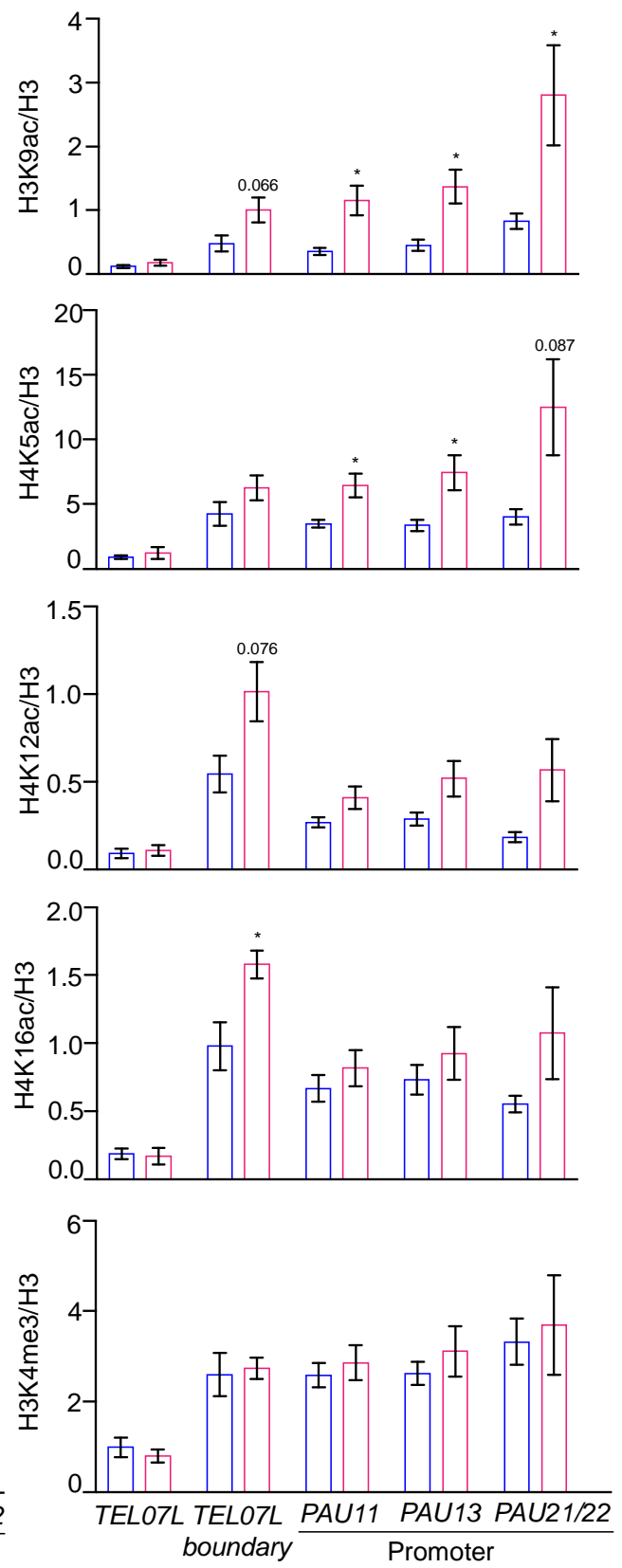

Figure 4: Histone acetylation increases at subtelomeric chromatin in set4 $\Delta$ cells in hypoxia.

chIP of H3K9ac, H4K5ac, H4K12ac, H4K16ac, and H3K4me3 at subtelomeric regions from wt (yEG001) and set4A (yEG322) strains grown to mid-log phase in YPD under 
aerobic (A) or hypoxic (B) conditions. Percent input of each acetyl or methyl mark are shown relative to percent input of total $\mathrm{H} 3$ levels. A minimum of three biological replicates are shown for histone mark chIPs and six biological replicates of histone H3 chIP was performed. The histone $\mathrm{H} 3$ immunoprecipitation is more efficient and consistent than histone $\mathrm{H} 4$, and therefore was used to normalize all of the histone modifications tested to total histone levels. For all panels, error bars indicate S.E.M. and asterisks represent $p$ values as calculated by unpaired t tests $\left({ }^{*}<0.05,{ }^{* *}<0.01,{ }^{* * *}\right.$ $<0.001)$. 
Figure 5

A

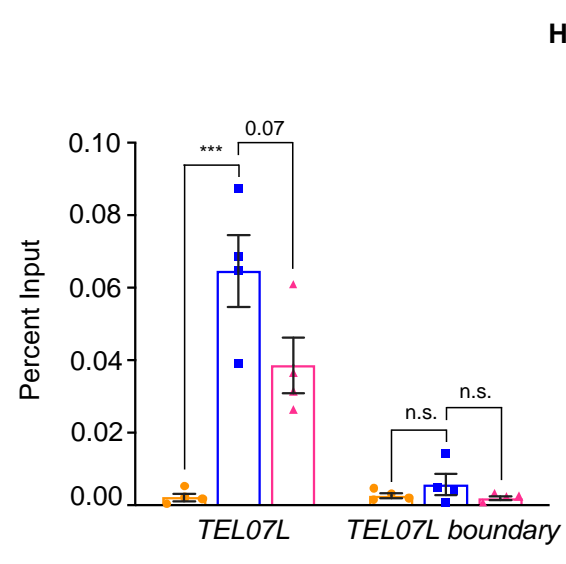

Hypoxic

- WT

- SIR3-HA

$\triangle \operatorname{set} 4$ DI
B

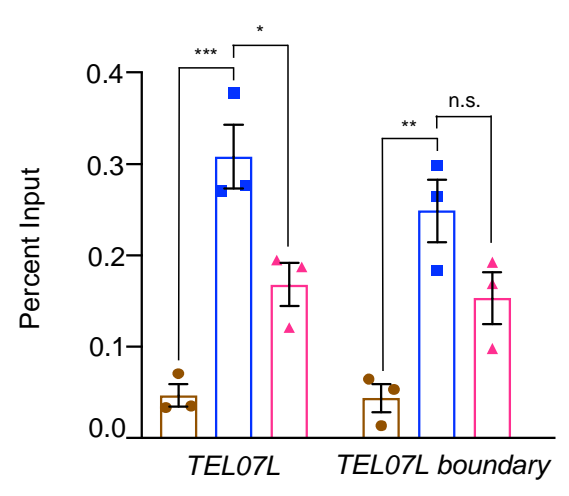

Hypoxic

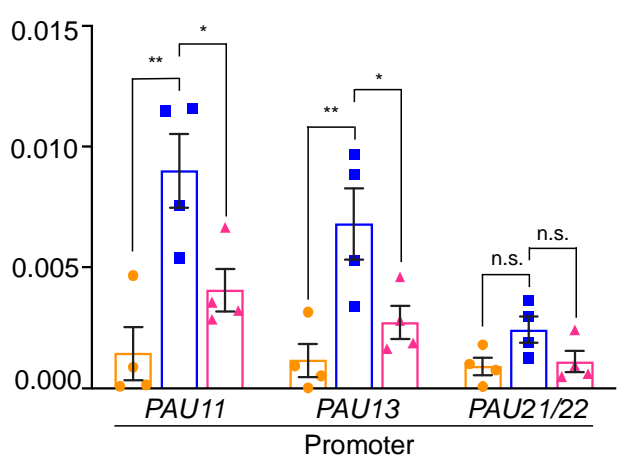

\section{Figure 5: Disrupted HDAC distribution at subtelomeric chromatin in the absence} of Set4.

A. chIP of wt (yEG001), Sir3-HA (yEG873) and set4A Sir3-HA (yEG874) strains grown to mid-log phase in YPD in hypoxic conditions. B. chIP of wt (yEG001), Rpd3-FLAG (yEG956) and Rpd3-FLAG set4A (yEG1010) strains grown to mid-log phase in YPD in hypoxic conditions. For both panels, percent input from at least three biological replicates is shown. The error bars indicate S.E.M. and asterisks represent $p$ values as calculated by one-way ANOVA and Tukey's post-hoc test $\left({ }^{*}<0.05,{ }^{* *}<0.01,{ }^{* *}<0.001\right)$. 
Figure 6

A

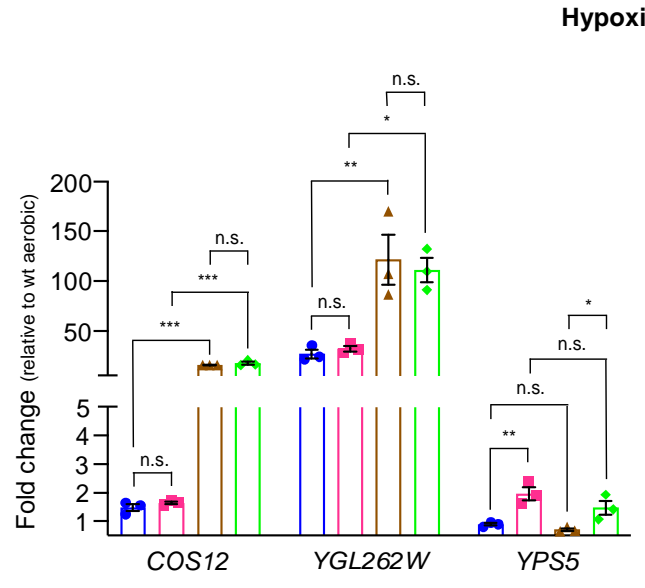

B

Hypoxic
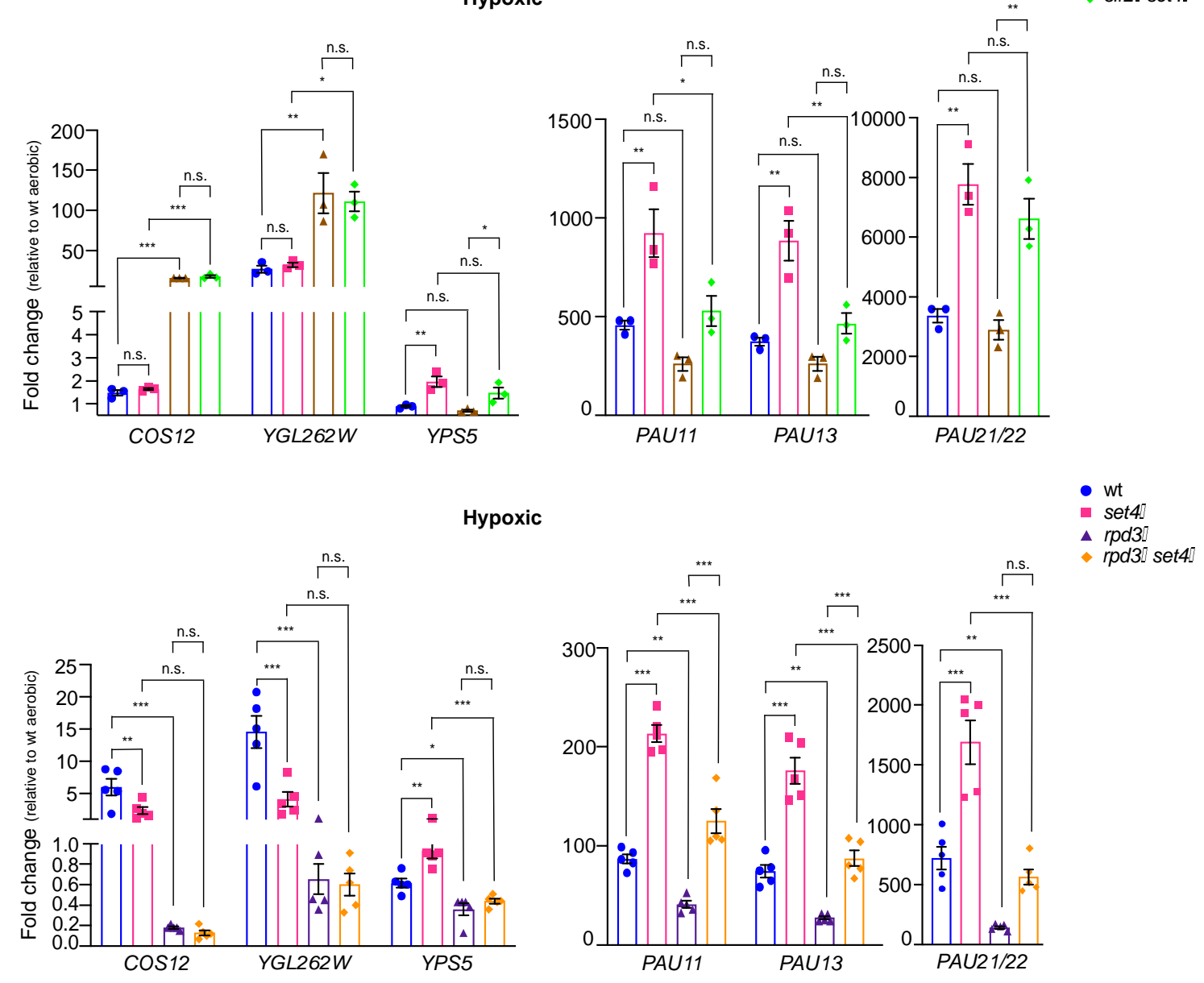

- set4]

$\Delta$ sir2]

sir2』 set4

Figure 6: Genetic interactions of Set4 with HDACs Sir2 and Rpd3 in regulating subtelomeric gene expression during stress.

A. RT-qPCR of subtelomeric genes from wt (yEG001), set4A (yEG1004), sir2 $\Delta$ (yEG1002) and set4A sir2 4 (yEG1005) strains grown under hypoxic conditions in YPD.

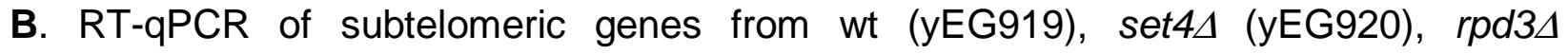
(yEG921) and set4A rpd34 (yEG922) strains grown under hypoxic conditions in YPD. For all experiments, expression levels were normalized to TFC1. Error bars represent S.E.M. from at least three biological replicates. For all panels, asterisks represent $p$ values as calculated by one-way ANOVA and Tukey's post-hoc test $\left({ }^{*}<0.05,{ }^{* *}<0.01\right.$, $\left.{ }^{* * *}<0.001\right)$. 
Figure 7

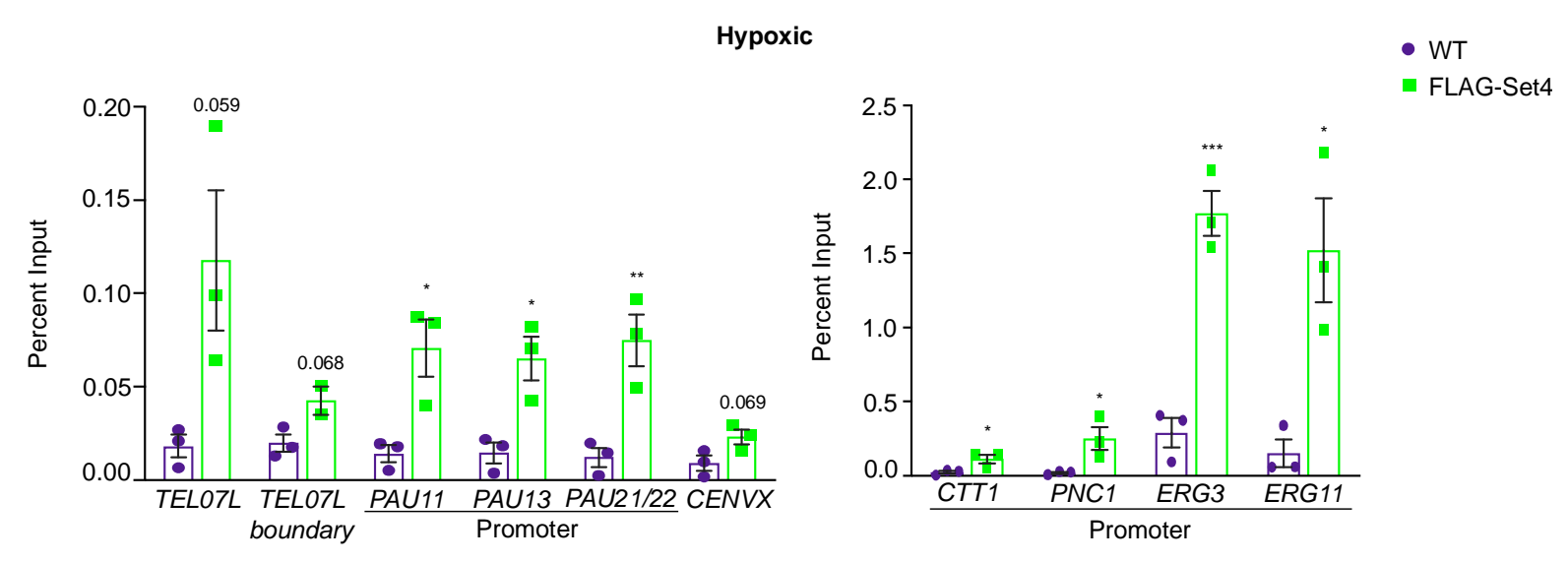

Figure 7: Set4 localizes to subtelomeric chromatin during stress.

chIP of FLAG-Set4 from cells grown under hypoxic conditions. Percent input from three biological replicates is shown. Left graph shows regions tested here for histone acetylation levels and HDAC binding. Right graph shows promoter regions previously identified as binding locations in under stress conditions $(10,14)$. Error bars represent S.E.M. and asterisks represent $p$ values as calculated by an unpaired $t$ test $\left({ }^{*}<0.05\right.$, $\left.{ }^{* *}<0.01,{ }^{* *}<0.001\right)$. 


\section{Figure 8}

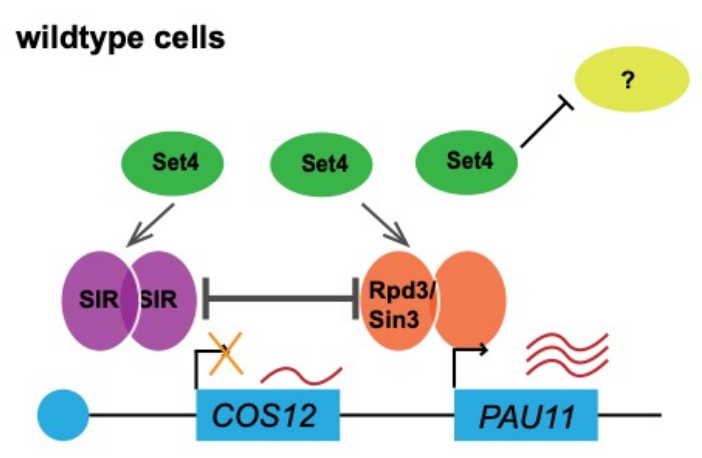

set4 $\Delta$ cells

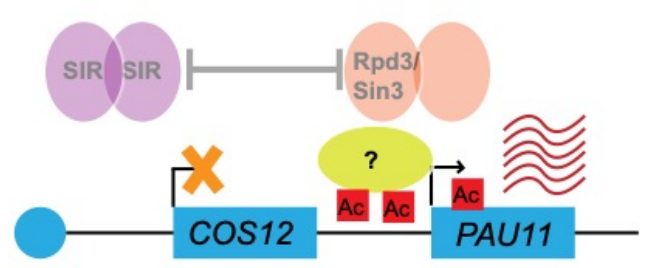

Figure 8: Model for Set4 function in subtelomeric gene regulation during hypoxic stress.

A partial depiction of TELOTL is shown indicating genes subject to telomere position effect (TPE) silencing, such as COS12, and genes repressed under standard growth conditions and induced in stress, such as PAU11. In wildtype cells, Set4 promotes the association of the SIR complex (Sir2/3/4) and Rpd3 with subtelomeric chromatin. The presence of these HDACs represses telomere-adjacent genes subject to TPE, such as COS12, and genes induced in limiting oxygen, such as PAU11. Set4, either alone or in cooperation with the SIR complex, Rpd3, or other yet unidentified chromatin regulators, may also inhibit the binding or activity of factors important for the positive regulation of stress response genes at subtelomeres (indicated by a question mark). In the absence of Set4, both the SIR complex and Rpd3 binding are diminished, resulting in increased histone acetylation and enhanced activation of PAU11 (and other PAU) genes. Genes subject to TPE, such as COS12, show enhanced repression upon loss of Set4, possibly due to diminishment of the antagonism between Rpd3 and the SIR complex, or due to compensation by other HDACs when Rpd3 and Sir2 levels are disrupted (56). This role for Set4 is most critical during stress, such as hypoxia when Set4 levels increase and it localizes to subtelomeric chromatin. Further genetic and physical interaction studies of Set4 at chromatin are likely to define the additional factors functioning with Set4, Rpd3 and the SIR complex in fine-tuning stress response genes within yeast subtelomeres. 


\section{References}

1. Jaiswal, D., Turniansky, R. and Green, E.M. (2017) Choose Your Own Adventure: The Role of Histone Modifications in Yeast Cell Fate. J Mol Biol, 429, 1946-1957.

2. Husmann, D. and Gozani, O. (2019) Histone lysine methyltransferases in biology and disease. Nat Struct Mol Biol, 26, 880-889.

3. Carlson, S.M., Moore, K.E., Green, E.M., Martín, G.M. and Gozani, O. (2014) Proteome-wide enrichment of proteins modified by lysine methylation. Nat Protoc, 9, 37-50.

4. Jethmalani, Y. and Green, E.M. (2020) Using yeast to define the regulatory role of protein methylation. Curr Protein Pept Sci, 21, 690-698.

5. Dillon, S.C., Zhang, X., Trievel, R.C. and Cheng, X. (2005) The SET-domain protein superfamily: protein lysine methyltransferases. Genome Biol, 6, 227.

6. Mas-Y-Mas, S., Barbon, M., Teyssier, C., Déméné, H., Carvalho, J.E., Bird, L.E., Lebedev, A., Fattori, J., Schubert, M., Dumas, C. et al. (2016) The Human Mixed Lineage Leukemia 5 (MLL5), a Sequentially and Structurally Divergent SET Domain-Containing Protein with No Intrinsic Catalytic Activity. PLoS One, 11, e0165139.

7. Tran, K. and Green, E.M. (2019) SET domains and stress: uncovering new functions for yeast Set4. Curr Genet, 65, 643-648.

8. Kim, T. and Buratowski, S. (2009) Dimethylation of H3K4 by Set1 recruits the Set3 histone deacetylase complex to 5' transcribed regions. Cell, 137, 259-272.

9. Pijnappel, W.W., Schaft, D., Roguev, A., Shevchenko, A., Tekotte, H., Wilm, M., Rigaut, G., Séraphin, B., Aasland, R. and Stewart, A.F. (2001) The S. cerevisiae SET3 complex includes two histone deacetylases, Hos2 and Hst1, and is a meiotic-specific repressor of the sporulation gene program. Genes Dev, 15, 2991-3004.

10. Tran, K., Jethmalani, Y., Jaiswal, D. and Green, E.M. (2018) Set4 is a chromatinassociated protein, promotes survival during oxidative stress, and regulates stress response genes in yeast. J Biol Chem, 293, 14429-14443.

11. Kemmeren, P., Sameith, K., van de Pasch, L.A., Benschop, J.J., Lenstra, T.L., Margaritis, T., O'Duibhir, E., Apweiler, E., van Wageningen, S., Ko, C.W. et al. (2014) Large-scale genetic perturbations reveal regulatory networks and an abundance of gene-specific repressors. Cell, 157, 740-752.

12. Tran, K. and Green, E.M. (2019) Assessing Yeast Cell Survival Following Hydrogen Peroxide Exposure. Bio Protoc, 9, e3149.

13. Lai, L.C., Kosorukoff, A.L., Burke, P.V. and Kwast, K.E. (2006) Metabolic-statedependent remodeling of the transcriptome in response to anoxia and subsequent reoxygenation in Saccharomyces cerevisiae. Eukaryot Cell, 5, 14681489.

14. Serratore, N.D., Baker, K.M., Macadlo, L.A., Gress, A.R., Powers, B.L., Atallah, N., Westerhouse, K.M., Hall, M.C., Weake, V.M. and Briggs, S.D. (2018) A Novel Sterol-Signaling Pathway Governs Azole Antifungal Drug Resistance and Hypoxic Gene Repression in. Genetics, 208, 1037-1055. 
15. De Las Peñas, A., Juárez-Cepeda, J., López-Fuentes, E., Briones-Martín-DelCampo, M., Gutiérrez-Escobedo, G. and Castaño, I. (2015) Local and regional chromatin silencing in Candida glabrata: consequences for adhesion and the response to stress. FEMS Yeast Res, 15, fov056.

16. Brown, C.A., Murray, A.W. and Verstrepen, K.J. (2010) Rapid expansion and functional divergence of subtelomeric gene families in yeasts. Curr Biol, 20, 895903.

17. Ellahi, A., Thurtle, D.M. and Rine, J. (2015) The Chromatin and Transcriptional Landscape of Native Saccharomyces cerevisiae Telomeres and Subtelomeric Domains. Genetics, 200, 505-521.

18. Jezek, M. and Green, E.M. (2019) Histone Modifications and the Maintenance of Telomere Integrity. Cells, 8, 199.

19. Yu, Y., Zhou, H., Deng, X., Wang, W. and Lu, H. (2016) Set3 contributes to heterochromatin integrity by promoting transcription of subunits of Clr4-Rik1-Cul4 histone methyltransferase complex in fission yeast. Sci Rep, 6, 31752.

20. McElroy, K.A., Jung, Y.L., Zee, B.M., Wang, C.I., Park, P.J. and Kuroda, M.I. (2017) upSET, the Drosophila homologue of SET3, Is Required for Viability and the Proper Balance of Active and Repressive Chromatin Marks. G3 (Bethesda), 7, 625-635.

21. Rincon-Arano, H., Halow, J., Delrow, J.J., Parkhurst, S.M. and Groudine, M. (2012) UpSET recruits HDAC complexes and restricts chromatin accessibility and acetylation at promoter regions. Cell, 151, 1214-1228.

22. Wang, W., Chaturbedi, A., Wang, M., An, S., Santhi Velayudhan, S. and Lee, S.S. (2018) SET-9 and SET-26 are H3K4me3 readers and play critical roles in germline development and longevity. Elife, 7, e34970.

23. Harvey, Z.H., Chakravarty, A.K., Futia, R.A. and Jarosz, D.F. (2020) A Prion Epigenetic Switch Establishes an Active Chromatin State. Cell, 180, 928940.e914.

24. Alejandro-Osorio, A.L., Huebert, D.J., Porcaro, D.T., Sonntag, M.E., Nillasithanukroh, S., Will, J.L. and Gasch, A.P. (2009) The histone deacetylase Rpd3p is required for transient changes in genomic expression in response to stress. Genome Biol, 10, R57.

25. Ruiz-Roig, C., Viéitez, C., Posas, F. and de Nadal, E. (2010) The Rpd3L HDAC complex is essential for the heat stress response in yeast. Mol Microbiol, 76, 1049-1062.

26. Sertil, O., Vemula, A., Salmon, S.L., Morse, R.H. and Lowry, C.V. (2007) Direct role for the Rpd3 complex in transcriptional induction of the anaerobic DAN/TIR genes in yeast. Mol Cell Biol, 27, 2037-2047.

27. Zhou, J., Zhou, B.O., Lenzmeier, B.A. and Zhou, J.Q. (2009) Histone deacetylase Rpd3 antagonizes Sir2-dependent silent chromatin propagation. Nucleic Acids Res, 37, 3699-3713.

28. Radman-Livaja, M., Ruben, G., Weiner, A., Friedman, N., Kamakaka, R. and Rando, O.J. (2011) Dynamics of Sir3 spreading in budding yeast: secondary recruitment sites and euchromatic localization. EMBO J, 30, 1012-1026. 
29. Ai, W., Bertram, P.G., Tsang, C.K., Chan, T.F. and Zheng, X.F. (2002) Regulation of subtelomeric silencing during stress response. Mol Cell, 10, 12951305.

30. Longtine, M.S., McKenzie, A., Demarini, D.J., Shah, N.G., Wach, A., Brachat, A., Philippsen, P. and Pringle, J.R. (1998) Additional modules for versatile and economical PCR-based gene deletion and modification in Saccharomyces cerevisiae. Yeast, 14, 953-961.

31. Martín, G.M., King, D.A., Green, E.M., Garcia-Nieto, P.E., Alexander, R., Collins, S.R., Krogan, N.J., Gozani, O.P. and Morrison, A.J. (2014) Set5 and Set1 cooperate to repress gene expression at telomeres and retrotransposons. Epigenetics, 9, 513-522.

32. Jezek, M., Gast, A., Choi, G., Kulkarni, R., Quijote, J., Graham-Yooll, A., Park, D. and Green, E.M. (2017) The histone methyltransferases Set5 and Set1 have overlapping functions in gene silencing and telomere maintenance. Epigenetics, 12, 93-104.

33. Bolger, A.M., Lohse, M. and Usadel, B. (2014) Trimmomatic: a flexible trimmer for Illumina sequence data. Bioinformatics, 30, 2114-2120.

34. Dobin, A., Davis, C.A., Schlesinger, F., Drenkow, J., Zaleski, C., Jha, S., Batut, P., Chaisson, M. and Gingeras, T.R. (2013) STAR: ultrafast universal RNA-seq aligner. Bioinformatics, 29, 15-21.

35. Huber, W., Carey, V.J., Gentleman, R., Anders, S., Carlson, M., Carvalho, B.S., Bravo, H.C., Davis, S., Gatto, L., Girke, T. et al. (2015) Orchestrating highthroughput genomic analysis with Bioconductor. Nat Methods, 12, 115-121.

36. Efron, B. (2004) Large-scale simultaneous hypothesis testing: the choice of a null hypothesis. J Am Stat Assoc, 99, 96-104.

37. Efron, B., Turnbull, B., Narasimhan, B. and Strimmer, K. (2015).package "locfdr".

38. Benjamini, Y. and Hochberg, Y. (1995) Controlling the false discovery rate: a practical and powerful approach to multiple testing. J.R. Statist. Soc. B., 57, 289300.

39. Teste, M.A., Duquenne, M., François, J.M. and Parrou, J.L. (2009) Validation of reference genes for quantitative expression analysis by real-time RT-PCR in Saccharomyces cerevisiae. BMC Mol Biol, 10, 99.

40. Liesche, J., Marek, M. and Günther-Pomorski, T. (2015) Cell wall staining with Trypan blue enables quantitative analysis of morphological changes in yeast cells. Front Microbiol, 6, 107.

41. Jezek, M., Jacques, A., Jaiswal, D. and Green, E.M. (2017) Chromatin Immunoprecipitation (ChIP) of Histone Modifications from Saccharomyces cerevisiae. J Vis Exp, 130, 57080.

42. Liu, C.L., Kaplan, T., Kim, M., Buratowski, S., Schreiber, S.L., Friedman, N. and Rando, O.J. (2005) Single-nucleosome mapping of histone modifications in S. cerevisiae. PLoS Biol, 3, e328.

43. Meluh, P.B. and Broach, J.R. (1999) Immunological analysis of yeast chromatin. Methods Enzymol, 304, 414-430.

44. Zeng, P.Y., Vakoc, C.R., Chen, Z.C., Blobel, G.A. and Berger, S.L. (2006) In vivo dual cross-linking for identification of indirect DNA-associated proteins by chromatin immunoprecipitation. Biotechniques, 41, 694, 696, 698. 
45. Luo, Z. and van Vuuren, H.J.J. (2009) Functional analyses of PAU genes in Saccharomyces cerevisiae. Microbiology (Reading), 155, 4036-4049.

46. Rachidi, N., Martinez, M.J., Barre, P. and Blondin, B. (2000) Saccharomyces cerevisiae PAU genes are induced by anaerobiosis. Mol Microbiol, 35, 14211430.

47. Kwast, K.E., Lai, L.C., Menda, N., James, D.T., Aref, S. and Burke, P.V. (2002) Genomic analyses of anaerobically induced genes in Saccharomyces cerevisiae: functional roles of Rox1 and other factors in mediating the anoxic response. $J$ Bacteriol, 184, 250-265.

48. Bendjilali, N., MacLeon, S., Kalra, G., Willis, S.D., Hossian, A.K., Avery, E., Wojtowicz, O. and Hickman, M.J. (2017) Time-Course Analysis of Gene Expression During the Saccharomyces cerevisiae Hypoxic Response. G3 (Bethesda), 7, 221-231.

49. Aguilar-Uscanga, B. and François, J.M. (2003) A study of the yeast cell wall composition and structure in response to growth conditions and mode of cultivation. Lett Appl Microbiol, 37, 268-274.

50. Deliu, E., Arecco, N., Morandell, J., Dotter, C.P., Contreras, X., Girardot, C., Käsper, E.L., Kozlova, A., Kishi, K., Chiaradia, I. et al. (2018) Haploinsufficiency of the intellectual disability gene SETD5 disturbs developmental gene expression and cognition. Nat Neurosci, 21, 1717-1727.

51. Wang, Z., Hausmann, S., Lyu, R., Li, T.M., Lofgren, S.M., Flores, N.M., Fuentes, M.E., Caporicci, M., Yang, Z., Meiners, M.J. et al. (2020) SETD5-Coordinated Chromatin Reprogramming Regulates Adaptive Resistance to Targeted Pancreatic Cancer Therapy. Cancer Cell, 37, 834-849.e813.

52. Ehrentraut, S., Weber, J.M., Dybowski, J.N., Hoffmann, D. and EhrenhoferMurray, A.E. (2010) Rpd3-dependent boundary formation at telomeres by removal of Sir2 substrate. Proc Natl Acad Sci U S A, 107, 5522-5527.

53. Tung, S.Y., Lee, K.W., Hong, J.Y., Lee, S.P., Shen, H.H. and Liou, G.G. (2013) Changes in the genome-wide localization pattern of Sir3 in Saccharomyces cerevisiae during different growth stages. Comput Struct Biotechnol J, 7, e201304001.

54. Morano, K.A., Grant, C.M. and Moye-Rowley, W.S. (2012) The response to heat shock and oxidative stress in Saccharomyces cerevisiae. Genetics, 190, 11571195.

55. Hickman, M.J., Spatt, D. and Winston, F. (2011) The Hog1 mitogen-activated protein kinase mediates a hypoxic response in Saccharomyces cerevisiae. Genetics, 188, 325-338.

56. Thurtle-Schmidt, D.M., Dodson, A.E. and Rine, J. (2016) Histone Deacetylases with Antagonistic Roles in Saccharomyces cerevisiae Heterochromatin Formation. Genetics, 204, 177-190. 
bioRxiv preprint doi: https://doi.org/10.1101/2021.05.11.443697; this version posted May 18, 2021. The copyright holder for this preprint (which was not certified by peer review) is the author/funder. All rights reserved. No reuse allowed without permission. 